\title{
Facilitators and barriers to cervical cancer screening among 1.5 and 2 nd-Generation Vietnamese-American women
}

\author{
Tanya U Luu ${ }^{1}$, Quyen Tran², Mo-Kyung Sin ${ }^{3 *}$ and Kelly McBroom ${ }^{3}$ \\ ${ }^{1}$ Virginia Mason Medical Center, WA, USA \\ ${ }^{2}$ Community Health Center of Snohomish County - Everett Central Clinic, WA, USA \\ ${ }^{3}$ Seattle University College of Nursing, USA
}

\begin{abstract}
Although knowledge of cervical cancer screening and prevention has become more prevalent in recent years, Vietnamese-American women continue to be disproportionately affected by cervical cancer incidence. The purpose of this qualitative study was to explore the barriers and facilitators to cervical cancer screening among 1.5 and 2nd generation Vietnamese- American women between 21-30 years old. A convenience sample of 10 women, identified as either 1.5 or 2nd generation Vietnamese-American, Washington State residents, aged 23-29 were recruited for telephone interviews. Content analysis was utilized for data analysis. Facilitators of cervical cancer screening included recommendation by primary care providers, openness to discussion with providers about gynecological issues, and participants' positive attitudes toward prevention. Barriers identified were a lack of knowledge, lack of recommendation by primary care providers, lack of health care coverage, lack of perceived risk, fear or discomfort associated with pap smears, and stigma related to sexual health. This exploratory study was the first study to specifically target a sub-group of young Vietnamese-American women of 1.5 and 2nd generation immigration status. Despite having the lowest cervical cancer screening rates across all age groups, this sub-group has been absent from previous studies on the subject of beliefs and behaviors surrounding cervical cancer and Vietnamese-American women. Knowledge gained from this study serves to enhance providers' knowledge in working with this sub-group. The insight gained can help to design more effective and culturally-sensitive interventions to address this disparity.
\end{abstract}

\section{Introduction}

Despite knowledge of cervical cancer screening and prevention becoming more widespread in recent years, one demographic in particular continues to experience a major disparity with cervical cancer incidence. Vietnamese-American women are disproportionately affected by cervical cancer compared to other ethnic and racial groups. According to early national data from the US Cancer Registry, cervical cancer prevalence in Vietnamese-Americans is the highest of any racial group (43 per 100,000); it is four times higher than all of the Asian groups combined and is more than double that of non-Hispanic white women $[1,2]$. It is not surprising that cervical cancer screening rates among Vietnamese- American women are among the lowest in the country. Researchers reported that only $55 \%$ of Vietnamese-American women had received a recent pap smear within the past 3 years [3]. This falls significantly short of the Healthy People 2020 goal for a cervical cancer screening rate of $93 \%$. For comparison, the overall screening rate among Asian-Americans is $70 \%$ and for non-Hispanic whites is $81 \%$ [4].

Factors that affect screening rates include knowledge of cervical cancer and human papilloma virus (HPV), traditional and cultural beliefs, and access to health care [5]. Additionally, it was found that the more acculturated the person (particularly with regard to cultural assimilation, education, and access to care), the likelier it was that the person would receive regular pap smears [6]. However, this does not explain why young Vietnamese-American women - who are typically more acculturated than their older counterparts are actually experiencing the lowest screening rates compared to other age groups. In general, screening rates among young women in the U.S. are very low; in King County, the rate among women 21 to 30 years old who have not received a pap smear within 3 years was $32 \%$, while women 25 to 44 years old and women 45 to 65 years old had rates of $12 \%$ and $15 \%$ respectively [7]. One study on U.S. women 17 and 30 years of age found that only $32 \%$ of Asian-American subjects had ever had a Pap test as compared to $70 \%$ of Caucasian women at the same campus, and Vietnamese-American women in particular experienced the lowest screening rate [8]. This is a concern because Human Papillomavirus (HPV) typically takes 10-15 years to develop into a malignancy, and the greatest number of women diagnosed with cervical cancer is between the ages of 30-49. Vietnamese-American women also experience the worst prognoses due to only being diagnosed in later stages [9].

Young Vietnamese-American women - particularly of 1.5 and 2nd generation immigration status - have historically been absent from studies surrounding cervical cancer. "1.5 generation immigrants" is a term used to describe people who immigrated to the United States as children and adolescents [10]. The experiential changes are influential to the process of defining one's identity [11]. The experience of 1.5 generation individuals is unique in the sense that their identity is split. 1.5 generation individuals live between two cultures; they can be cultural brokers in their own homes but can also be full participants of the adoptive culture of contemporary Western context $[10,12,13]$. Their

${ }^{\star}$ Correspondence to: Mo-Kyung Sin, Seattle University College of Nursing, 901 12th Ave P.O. Box 222000 Seattle, WA 98122-1090, E-mail: sinm@seattleu.edu

Key words: barriers, cervical cancer screening, facilitators, Vietnamese American women

Received: February 28, 2020; Accepted: March 18, 2020; Published: March 25, 2020 
varying American/immigrant identities are a result of the acculturation process to the mainstream culture and their foreign-born upbringing. This is in stark contrast with their 1st generation parents (who might identify solely with their cultures of origin) or their American-born siblings/peers (who might identify solely as American). 2nd generation specifically refers to people who were born in the United States to at least one 1st generation (immigrant) parent [14]. Though their birth status should grant 2 nd generation individuals a more solid identity as American, most identify strongly with their ancestral origins [14]. Because they are raised by 1st generation parents, they too might live between two cultures and struggle to balance conflicting family and social expectations [15].

Nearly all studies on cervical cancer screening behaviors among Vietnamese-American women have focused primarily on people who were over 40 years old, married, or born in Vietnam $[2,16]$. Thus, it would be worthwhile to explore the factors affecting the ability of young Vietnamese-American women - particularly of 1.5 and 2nd generation immigration status - to receive cervical cancer screening since they experience the world differently than their immigrant parents and older counterparts, which could shape their health behaviors. For example with regards to mental health, 1.5 and 2nd generation VietnameseAmericans are less likely to seek out help due to stigma and fear of bringing shame to their families $[17,18]$. Similarly, in Vietnamese culture, sexual activity before marriage and even just discussion about the topic of sexuality is generally discouraged [19]. Considering that many Vietnamese-American women correctly associated sexual activity with increased risk for HPV transmission, it would be appropriate to infer that young Vietnamese-American women are not getting screened for cervical cancer due to similar fears of stigma and shame $[1,20]$. Sexuality might be only one of many factors that influences cervical cancer screening rates among young Vietnamese-American women; it would be worthwhile to explore attitudes and perceptions of 1.5 and 2nd generation Vietnamese- American women as they navigate cervical cancer screening while balancing the expectations of their culture, families, and society. Thus, the purpose of this study is to explore the facilitators and barriers to receiving recommended cervical cancer screening among 1.5 and 2nd generation Vietnamese-American women.

\section{Methods}

\section{Study design}

A qualitative participatory research study using individual telephone interviews was conducted to explore cervical cancer screening barriers and facilitators. This project was conducted in the Seattle area of Washington from September 2017 to June 2018. It was approved by the University's Institutional Review Board, and verbal informed consent was obtained in English from each participant via telephone.

\section{Conceptual framework}

Modeled after the success of Sin and colleagues' study in 2016, which similarly explored the barriers and facilitators to cancer screening among a specific ethnic sub-group, the Health Behavior Framework (HBF) was utilized as the theoretical framework in this study [21,22]. The HBF has widely served as a theoretical approach in several studies among Asian American sub-group due to its unique incorporation of cultural influence into the examination of behaviors [21-24]. The HBF integrates theoretical formulations from several major theories which explore variables that impact an individual's health behavior.
The domains embedded in the HBF include individual variables (e.g., knowledge, health beliefs,

past health behaviors, cultural factor and beliefs), the provider and health care system factors (e.g., provider characteristics, communication style, health care setting, and practice patterns), health insurance coverage, and societal norms [21,22,25-28].

\section{Sample and Setting}

In this study, we recruited Vietnamese American women via wordof-mouth and a convenience snowball sampling method. The inclusion criteria of the study were: 1) female, 2) age 21-30,3) identified as Vietnamese American, 4) currently residing in Washington State. Women who have had a history of hysterectomy or those who have attended educational classes on cervical cancer over the last 3 to 5 years were excluded from the study. Since both of the principle investigators are Vietnamese American, we utilized our existing networks of friends and families for introduction and referrals. When a woman was approached for recruitment, she was provided with an overview of the study and information on the use of audio-recordings for individual interviews. Once potential participants expressed interest in participating in the study, the principal investigator (T.L.) obtained her phone number and arranged for the phone interview. None of the participants were known to or had any relationship with our research team prior to the study.

\section{Data collection procedure}

Following approval by the Seattle University Institutional Review Board, subjects were interviewed via telephone using a semi-structured questionnaire developed by the researchers. The interview guide was developed by both principle investigators and reviewed by a researcher with expertise in qualitative and cancer prevention studies. The items in the interview guide were modeled after a qualitative study conducted by our colleagues [22] and developed based on the HBF domains.

Interviews were conducted over the phone by two PIs (T.L. and Q.T.). They are both identified as Vietnamese-American, pursuing a career in healthcare field, and are familiar with Vietnamese culture, and cervical cancer-related issues. All interviews were conducted in English. Each interview lasted for approximately 15 to 30 minutes. Subjects were told that phone conversations would be recorded but all of the conversational exchanges over the phone would be kept confidential. They were informed that all information gathered in this study would be used for research and educational purposes only; their names and identifying information would not be revealed (we did not collect any direct identifying information in this study). Demographic information (e.g., age, education status, and length of time living in the United States) were collected to better understand the participants' characteristics. The PIs addressed the study background, confidentiality, and stated that there would be no right or wrong answers at the beginning of each interview. All participants were informed that they can decline to answer specific questions or terminate their study participation at any time. Audio from the interviews were recorded and stored on a password protected computer. Immediately after each interview, we conducted transcription for data analysis.

\section{Results}

\section{Demographic characteristics}

With the exception of one subject, all were born outside of the United States. The average age of the participants was 27 , and the 
average length of time living in the United States was 15.9 years. Most of the study participants had some type of health care coverage, and the majority had completed a bachelor's degree.

\section{Facilitators}

Facilitators identified to receive cervical cancer screening among the participants included recommendation by a primary care provider, openness to discussion with the provider about gynecological issues, and participants' positive attitudes toward prevention. Relevant details and significant quotes from the participants are provided below.

\section{Recommendation by provider}

Most of the participants who had received a pap smear in the past were recommended to do so by their primary care providers. Many expressed that they would rely primarily on their providers for knowledge and recommendations regarding any health issues, and they would prefer for their providers to remind them of any routine exams.

"I'm not the doctor. I don't know if I would need to do certain things. I need to ask her to see if I need it."

"I always get notified about [getting a pap smear], so I just trust my doctor to send me the appointment reminder."

"Because my family doctor wanted me to [get a pap smear]."

"Just by going to the doctor's office [I obtained this information about cervial cancer]."

\section{Openness to discussion with provider about gynecological concerns}

All of the participants expressed that they were comfortable speaking to their primary care providers. However, it was evident that most of the participants would only be comfortable talking about gynecological health or receiving a pap smear from a female provider.

“... when you have gynecological concerns, you would request a female provider because they are shy. If female providers are not available, then the women would feel shy and embarrassed with a male provider and feel uncomfortable, and they are not open-minded when it comes to getting the procedure."

“... with a female, then I would be totally fine. You know what it is. Gender difference. And since I am Vietnamese... it is a little bit related. I would be a little bit uncomfortable if it is a male doctor... I would say that is the biggest problem that [Vietnamese women] would not ask their male doctors or talk about their private parts. They would think it is the cherished part. 'It is only my husband who knows and you cannot know unless you are [a woman]."

\section{Positive attitudes toward prevention}

Participants who had been screened for cervical cancer in the past were generally more proactive about their health and checked in with their primary care providers regularly.

Participants who had not received pap smears indicated that being invested in and proactive about one's health should drive someone to receive a pap smear.

"Yes, [I had a pap smear in the past]." "Just a general check-up. I just wanted to make sure that I am okay, that everything is okay."

"Being aware of what health issues might arise down the road in your lifetime if things are not checked. So being educated about it and making it the norm for ladies who are Vietnamese-American to go and get that checked out at a regular basis..."

"If it is for my health, I would totally ask my doctor if I need to [get a pap smear]."

\section{Barriers}

Several barriers to cervical cancer screening among 1.5 and 2nd generation Vietnamese- American women were identified. These included lack of knowledge, lack of recommendation by a provider, lack of health care coverage, lack of perceived risk, fear or discomfort associated with pap smears, and stigma related to sexual health Lack of knowledge

The greatest barrier to receiving a pap smear among the participants was a lack of knowledge about cervical cancer. The first area of knowledge that was lacking encompassed the basic characteristics of cervical cancer - what it is, what causes it, and how it manifests and presents itself.

"I just heard the name, but not really detailed information. I do not know anything about it. I understand it is a type of cancer. But I do not know what a cervix is."

"No, I know nothing. I know cancers, but I do not know specific kinds of cancers. I mean, cancers do stuff to your body. Can you tell me what is it about?"

Most of the participants were not aware of methods of prevention. Some were completely unaware or confused about the purpose and process of receiving a pap smear, and did not equate pap smears with cervical cancer screening. For example, when asked if her provider had ever recommended for her to be screened for cervical cancer, one participant responded:

"That I do not remember. But probably when they did the pap smear. They just suggested to do the pap smear to test to see if it is normal or not, but they did not mention about the cervical cancer screening."

"I never thought of doing the screening, and my provider never talked to me about it. We never had a discussion about this type of screening in the past."

\section{Lack of provider recommendation}

As seen by the theme of lack of knowledge above, not all health care providers had recommended their clients to get a pap smear. Only half of the participants had been recommended by their providers to be screened for cervical cancer.

\section{Lack of health care coverage}

Neither of the two participants without health care coverage had received a pap smear. Participants shared lack of health care coverage as a barrier for cervical cancer screening.

"And for those who do not have health insurance, then they would have to pay for it, so sometimes, there are charity that they can pay for you. But when they do not have insurance, they then would have to pay a lot out of pocket."

"We have more motivation because here we have health insurance and easier access to health service. People who have health insurance, it is easier for them, because they have support." 


\section{Lack of perceived risk}

All participants believed they had either a lower or equal risk of contracting cervical cancer compared to others in their age group. When asked about potential barriers to screening, many participants expressed that being screened is not imperative unless a person is symptomatic or if they have had multiple sex partners.

"... they also think in their head that if it is a disease, then symptoms will show and appear. If they feel like they do not have anything, then they would not go get check-ups." "For example, like my mom, until it happens then she would [not] freak out about it. But regularly, they do not really care. They do not really think it is important because they do not know anything about it."

"Because I do not need it yet. It is more needed for women who are active in sex or more highly active in sex."

"Because I do not think I need [a pap smear]. I kind of know that if I am not actively having sex, then I do not need it personally."

\section{Fear/discomfort with pap smears}

The participants who had received pap smears before generally had no issues with the procedure. However, many of those who had not received pap smears before indicated a fear of discomfort or pain associated with the procedure.

"Because when the women hear about the fact that they have to spread their vagina for the procedure, they are already scared. Sounds like you are giving birth. Like for me, if I hear about what I have to during the procedure, then I feel really scared already. If they do not have to spread it out like that, or they can give us a test for us just to pee out. Something less invasive and easier. Or they can give out anesthesia during the procedure. Because it will be difficult. Like for people like myself, I am extremely scared of pain, that is why it is very difficult."

"My doctor tried it before, but I thought it was painful, so I did not want to do it. So, then I gave up."

"Some people might have heard that it will bring a lot of discomfort."

\section{Stigma related to sexual health}

Some of the participants believed that cultural stigma regarding sexual health concerns greatly limits 1.5 and 2 nd generation Vietnamese-American women's ability to openly discuss the issues and seek care.

"I am aware how Vietnamese are, you know. Culturally, we do not want to talk about sex. We do not talk about diseases that are related to sex... Culturally, we believe that you are not supposed to have any sexual activity until you are married. So, let's say young girls, if they are sexually active, they would not want to talk to their parents about it. So, if there is not anyone to tell them, "Oh, you are sexually active. You should get tested," and stuff, at a young age, they are not aware of that and do not know the risk that comes with being sexually active."

"So my mom has always taught me to never get a pap smear. 'Do not do that because that is going to ruin your virginity, like that. That is ridiculous."

\section{Discussion}

We had previously speculated that cultural beliefs and tradition driven stigma surrounding sexual health would be the most significant barriers to being screened for cervical cancer among 1.5 and 2 nd generation Vietnamese-American women. However, although a few participants had mentioned the lack of open discussion regarding anything related to sexual health, it was the general lack of knowledge and misinformation about cervical cancer that seemed to be the primary obstacles to cervical cancer screening.

Firstly, there was a significant knowledge gap regarding basic information about cervical cancer - what it is, what causes it, and how it presents itself. These findings are consistent with previous studies conducted on Vietnamese-American women [2,5]. The majority of the participants had little to no knowledge of cervical cancer, and a significant number of them required additional explanation about the anatomy of the cervix. A majority of the participants correctly associated sexual activity with increased risk for developing cervical cancer. However, a majority was unaware of the human papillomavirus, how it is the greatest contributor to the development of cervical cancer, and that there is a vaccine available for it.

Additionally, all the participants were under the impression that cervical cancer manifests in obvious symptoms, such as pelvic pain, abnormal bleeding, or foul discharge, when in fact, early cervical cancer may not cause any signs and symptoms at all [29]. Worse still, many of the signs and symptoms exhibited during later stage cervical cancer mimic those experienced during a woman's period and may be hard to differentiate [29]. This belief is troubling because many of the participants expressed that they, like many others within their community, would likely not seek medical attention unless they were symptomatic.

There was also a surprising amount of confusion about pap smears. While a majority of participants indicated that they knew what a pap smear is, they only had a minimal understanding of what it is, how it is performed, and what exactly is being tested by the procedure. Most remarkably, a few did not equate the phrase "pap smear" with "cervical cancer screening", even though they had specified that they knew what a pap smear was. A few participants who previously reported that they had received a pap smear, later made remarks that they had not been screened for cervical cancer. There is a concern that because they do not identify pap smears as a method to prevent something as serious as cervical cancer, receiving pap smears is not imperative for them. Because many of the participants said that they, like many within their community, gained their health knowledge from their friends and family, it is likely that this belief is pervasive and is contributing to the low rate of cervical cancer screening within this community.

It is also likely that the low sense of urgency to be screened is driven by a lack of perceived risk. The study participants were generally unaware that Vietnamese-Americans experience a major disparity when it comes to cervical cancer incidence when compared to other racial and ethnic groups [2]. Additionally, some of the participants felt it was unnecessary to be screened based on their sexual history, particularly those who have never been sexually active or who have only had one sexual partner in their lifetime. Although this group technically experiences a lower risk of contracting HPV compared to others who have had multiple lifetime sexual partners, it is still an official recommendation by the CDC to be screened, regardless of sexual history [29].

Because participants indicated that they rely on their providers and their networks for information, the responsibility of health education ultimately falls on the providers. Nearly half of the participants were not recommended to receive a pap smear by the providers even though all met the minimum requirements to be screened, as recommended by the CDC [29]. Since discussion about sexual health is stigmatized by 
the community and since incorrect information is surely circulating, it is a serious obligation for providers within this community to provide unbiased, unadulterated, evidence-based recommendations and knowledge to their patients, and to emphasize the gravity of the situation so that patients can make timely, educated health decisions to ensure a long life of good health.

\section{Limitations}

Despite having a small sample size in this study, the emergence of recurring themes in our data analysis led us to believe that data saturation was achieved. As the study specifically targeted 21-30 years old Vietnamese American women living in the Seattle area, future studies could benefit from other Vietnamese Americans living in different geographical areas of Washington State and the US as a whole. An overwhelming $90 \%$ of the recruited participants are identified as 1.5 generation Vietnamese American while the rest is identified as $2^{\text {nd }}$ generation Vietnamese American (10\%). Since the study aims to explore the unique perspectives of both subgroups of 1.5 and $2^{\text {nd }}$ generation Vietnamese Americans, having more participants who are identified as $2^{\text {nd }}$ generation would allow for increased diversity in the heterogeneity and representativeness of the participants.

A limitation of the study was the lack of standardization in the interviewing process and in the delivery of the health education component of the phone interview encounter. Despite having a structured interview guide, each PI had a different approach in asking questions and in providing the educational aspect of cervical cancer screening among their participants. This variation could subsequently have led to a difference in the ability of the PIs to evoke rich and meaningful responses from participants. Furthermore, the interview process revealed that a couple questions (i.e. "How big of a role does your Vietnamese culture factor into your life?”) were deemed as vague, lacking in context, and needed re-wording or clarification from PIs. One pitfall of the interview guide was a question that specifically asked about the HPV vaccine. This question was raised under the assumption that participants have had prior knowledge of the vaccine and its role in cervical cancer screening. On the contrary, a majority of participants had no knowledge about this vaccine and struggled to provide an accurate response.

A participant's profession could largely influence the extensiveness of her health knowledge and health prevention awareness, such as nursing, pharmacy, or medicine. This data would allow for better comparison against those whose profession is not related to healthcare. Thus, participant's occupation can be obtained in future studies. In addition, information on the participant's previous exposure to annual wellness check-up can be obtained since that serves as a potential facilitative health behavior of cervical cancer screening.

\section{Conclusion}

Although knowledge of cervical cancer screening and prevention has become more prominently disseminated in recent years, Vietnamese American women continue to be disproportionately affected by cervical cancer incidence. Our exploratory study is the first study to specifically target a sub-group of young Vietnamese-American women, particularly of 1.5 and 2nd generation immigration status. Despite having the lowest cervical cancer screening rates across all age groups, this sub-group has been neglected from previous studies on cervical cancer screening behaviors among Vietnamese-American women. While it is important that health care providers continue to strive to promote cervical cancer screening in each preventative health visit, more specific targeted strategies are needed to promote the beneficial significance of cervical cancer screening among young Vietnamese-American.

In recent years, multifaceted and culturally appropriate interventions have emerged as effective strategies to address health disparities among ethnic groups. In their community-based intervention trial, Ma and colleagues [1] found a significantly higher cervical cancer screening rate among Vietnamese American women when they had an opportunity to participate in culturally relevant group education, visual aids and materials, patient navigation, clientphysician intervention video, as well as referral and reminder. Similarly, underserved Korean women also benefited from a community-based intervention and multifaceted modality strategies when combine psychoeducational counseling and community accessible programs with patient navigation health educations and services in the promotion of cervical cancer screening [30]. Tailored interventions entailing telephone counseling, in-person counseling, and physician letters have also been found to be effective in promoting screening behavior among non-adherent women [31]. Taking into consideration the success of these multifaceted and culturally-tailored interventions and the unique dynamics role of culture, families, friends, and health beliefs that shape the health behaviors of young Vietnamese American women, more studies and intervention programs should be designed to better help serve and lower the disparity in this sub-group.

\section{References}

1. Ma GX, Fang C, Tan Y, Feng Z, Ge S, et al. (2015) Increasing Cervical Cancer Screening Among Vietnamese Americans: A Community-Based Intervention Trial Journal of Health Care for the Poor and Underserved 26: 36-52. [Crossref]

2. Ma GX, Gao W, Fang CY, Tan Y, Feng Z, et al. (2013) Health beliefs associated with cervical cancer screening among Vietnamese Americans. Journal of Women's Health 22: 276-288. [Crossref]

3. Taylor V, Yasui Y, Nguyen T, Woodall E, Do H, et al. (2009) Pap smear receipt among Vietnamese immigrants: The importance of health care factors. Ethnicity \& Health 14 575-589. [Crossref]

4. Wang J, Sheppard V, Schwartz M, Liang W, Mandelblatt J (2008) Disparities in cervical cancer screening between Asian American and Non-Hispanic White women. Cancer Epidemiology Biomarkers \& Prevention 17: 1968-1973. [Crossref]

5. Do M (2015) Predictors of cervical cancer screening among Vietnamese American women. Journal of Immigrant and Minority Health, 17: 756-764. [Crossref]

6. Nguyen AB, Clark TT (2014) The role of acculturation and collectivism in cancer screening for Vietnamese American women. Health Care for Women International 35 : 1162-1180. [Crossref]

7. Public Health - Seattle \& King County (2014) Cervical Cancer Screening Guidelines Not Met (Females Age 21-65) Seattle, WA.

8. Tang TS, Solomon LJ, Yeh CJ, Worden JK (1999) The role of cultural variables in breast self-examination and cervical cancer screening behavior in young Asian women living in the United States. Journal of Behavioral Medicine 22: 419-436. [Crossref]

9. Ma G, Toubbeh J, Wang M, Shive S, Cooper L, et al. (2009) Factors associated with cervical cancer screening compliance and noncompliance among Chinese, Korean, Vietnamese, and Cambodian Women. Journal of the National Medical Association 101: 541-551. [Crossref]

10. Rojas L (2012) Gen 1.5: Where an immigrant generation fits in. Retrieved May 12 2018, from http://www.scpr.org/blogs/multiamerican/2012/03/21/7963

11. Phinney JS, Rotheram MJ (1987) Children's ethnic socialization: Pluralism and development. Newbury Park: Sage Publications.

12. Goldschmidt M, Miller C (2005) Beyond the academic needs of generation 1.5 . Research \& Teaching in Developmental Education, 21: 10-19.

13. Pham Bach (2012) Generational struggles and identity conflict among 1.5 generation Vietnamese Americans: Finding a middle ground (Master's Thesis, East Carolina University) Accessed from http://thescholarship.ecu.edu/handle/10342/3955 
14. Pew Research Center. Second-generation Americans - A Portrait of the adult children of immigrants. (2013, February 07) Accessed on May 12, 2018, from http://www. pewsocialtrends.org/2013/02/07/second-generation-americans/

15. Bersani B (2013) A Game of Catch-Up? The Offending Experience of SecondGeneration Immigrants. Crime \& Delinquency 60: 60-84. [Crossref]

16. Yoo GJ, Nhung Le M, Vong S, Lagman R, Lam AG (2011) Cervical cancer screening: Attitudes and behaviors of young Asian American women. Journal of Cancer Education: The Official Journal of the American Association for Cancer Education 26: 740-746. [Crossref]

17. Augsberger A, Yeung A, Dougher M, Hahm HC (2015) Factors influencing the underutilization of mental health services among Asian American women with a history of depression and suicide. BMC Health Services Research, 15: 542. [Crossref]

18. Lee S, Juon H, Martinez G, Hsu C, Robinson E, et al. (2008) Model minority at risk: Expressed needs of mental health by Asian American young adults. Journal of Community Health 34: 144-152. [Crossref]

19. Gor BJ, Chilton JA, Camingue PT, Hajek RA (2011) Young Asian Americans' knowledge and perceptions of cervical cancer and the human papillomavirus. Journal of Immigrant and Minority Health 13: 81-86. [Crossref]

20. Marlow LAV, Waller J, Wardle J (2015) Barriers to cervical cancer screening among ethnic minority women: A qualitative study. The Journal of Family Planning and Reproductive Health Care 41: 248-254. [Crossref]

21. Bastani R, Glenn B, Taylor V, Chen MS, Nguyen T, et al. (2010) Integrating theory into community interventions to reduce liver cancer disparities: The health behavior framework. Preventive Medicine 50: 63-67. [Crossref]

22. Sin M, Ha A, Taylor V (2016) Sociocultural barriers to lung cancer screening among Korean immigrant men. Journal of Community Health 41: 790-797. [Crossref]
23. Glenn B, Bastani R, Reuben D (2006) How important are psychosocial predictors of mammography receipt among older women when immediate access is provided via onsite service?. American Journal of Health Promotion 20: 237-246. [Crossref]

24. Taylor V, Choe J, Yasui Y, Li L, Burke N, Jackson J (2005) Hepatitis B awareness, testing, and knowledge among Vietnamese American men and women. Journal of Community Health 30: 477-490. [Crossref]

25. Babey S, Hastert T, Yu H, Brown ER (2008) Physical activity among adolescents. When do parks matter?. American Journal of Preventative Medicine 34: 345-348. [Crossref]

26. Ponce N, Huh S, Bastani R (2005) Do HMO market level factors lead to racial/ethnic disparities in colorectal cancer screening? A comparison between high-risk Asian and Pacific Islander Americans and high-risk Whites. Medical Care 43: 1101-1108. [Crossref]

27. Wagner EH (1998) Chronic disease management: What will it take to improve care for chronic illness?. Effective Clinical Practice 1: 2-4. [Crossref]

28. Zapka JG, Lemon SC (2004) Interventions for patients, providers, and health care organizations. Cancer 101: 1165-1187. [Crossref]

29. Centers for Disease Control and Prevention. What are the symptoms of cervical cancer? (2017, February 13) Retrieved June 26, 2019, from https://www.cdc.gov/cancer/ cervical/basic info/symptoms.htm

30. Fang CY, Ma GX, Tan Y, Chi N (2007) A multifaceted intervention to increase cervical cancer screening among underserved Korean women. Cancer Epidemiology Biomarkers \& Prevention, 16: 1298-1302. [Crossref]

31. Champion V, Maraj M, Hui S, Perkins AJ, Tierney W, et al. (2003) Comparison of tailored interventions to increase mammography screening in nonadherent older women. Prev Med 36: 150-158. [Crossref]

Copyright: (C2020 Luu TU. This is an open-access article distributed under the terms of the Creative Commons Attribution License, which permits unrestricted use, distribution, and reproduction in any medium, provided the original author and source are credited. 